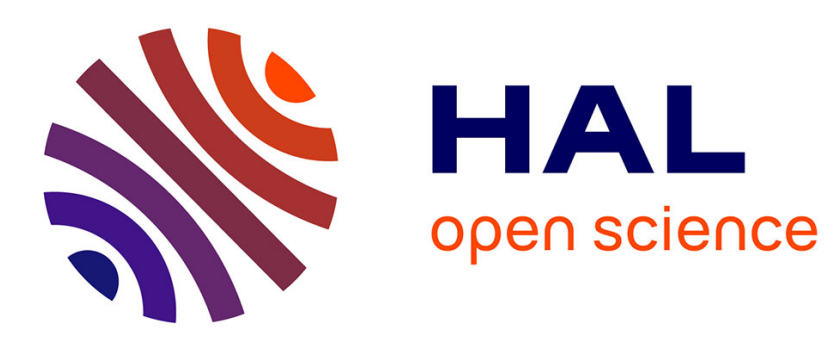

\title{
Awake mapping of the brain connectome in glioma surgery: Concept is stronger than technology
}

\author{
H. Duffau
}

\section{To cite this version:}

H. Duffau. Awake mapping of the brain connectome in glioma surgery: Concept is stronger than technology. EJSO - European Journal of Surgical Oncology, 2015, 41 (9), pp.1261-1263. 10.1016/j.ejso.2015.05.009 . hal-01985790

\section{HAL Id: hal-01985790 https://hal.science/hal-01985790}

Submitted on 18 Jan 2019

HAL is a multi-disciplinary open access archive for the deposit and dissemination of scientific research documents, whether they are published or not. The documents may come from teaching and research institutions in France or abroad, or from public or private research centers.
L'archive ouverte pluridisciplinaire $\mathbf{H A L}$, est destinée au dépôt et à la diffusion de documents scientifiques de niveau recherche, publiés ou non, émanant des établissements d'enseignement et de recherche français ou étrangers, des laboratoires publics ou privés. 


\title{
Awake mapping of the brain connectome in glioma surgery: Concept is stronger than technology
}

\author{
H. Duffau ${ }^{\mathrm{a}, \mathrm{b} ; *}$ \\ ${ }^{a}$ Department of Neurosurgery, Montpellier University Medical Center, Montpellier 34295, France \\ ${ }^{\mathrm{b}}$ Institute for Neuroscience of Montpellier, INSERM U-1051, Hopital Saint Eloi, Montpellier 34298, France
}

Keywords: Awake mapping; Brain Gliomas; Connectome; Surgery; Technology

Gliomas are the most frequent primary brain tumors and account for about half of all primary central nervous system neoplasms. They represent a major public health problem. Firstly, overall survival remains short, especially in glioblastomas (less than 12e18 months). Secondly, due to the infiltrative nature of gliomas, which usually migrate along the white matter tracts within the brain, patients may experience severe neurological deficits preventing to enjoy normal social and professional lives. Thirdly, the management of glioma patients is very expensive, in particular when these patients are not autonomous anymore.

Despite the lack of Phase III study, surgical resection is currently considered as the first treatment option, as recommended by the European guidelines. Indeed, a large amount of recent data supports the impact of surgery on the natural history of this disease, both in low-grade gliomas and highgrade gliomas. For example, the largest surgical series of diffuse low-grade gliomas ever reported (by the French Glioma Consortium), with more than one thousand patients who underwent maximal resection, showed that the overall survival was around 15 years ${ }^{1}$-versus about 6 years in classical non-surgical series. In addition, surgery may be useful to control intractable epilepsy and to decrease mass effect. Finally, it provides tumor sample to benefit from histomolecular examination and to select the best adjuvant therapeutic strategy, e.g. chemotherapy and/or radiotherapy.

\footnotetext{
* Department of Neurosurgery, Gui de Chauliac Hospital, CHU Montpellier, 80 Avenue Augustin Fliche, 34295 Montpellier, France. Tel.: p33 4673366 12; fax: p334673369 12.

E-mail address: h-duffau@chu-montpellier.fr
}

In this setting, glioma surgery is traditionally considered as a dilemma, due to the brain infiltration by tumoral cells, thus due to the risk of postoperative neurological worsening, which is higher when the extent of tumor removal is increased for oncological reasons. To overcome this problem, the vast majority of studies in the past decade focused on technical advances in neuroimaging into the operative theater. In particular, preoperative anatomic, metabolic and functional MR imaging has been incorporated in neuronavigation systems, to better define the surgical approach and the limits of resection. More recently, new devices based upon intraoperative MR imaging have been introduced.

However, in spite of these methodological developments based upon computer-aided surgery, and beyond the fact that these techniques are very expensive and not available in all departments of neurosurgery worldwide, no demonstrations have been made that the benefit-risk ratio of glioma surgery was significantly increased. Indeed, no series using such tools were able to demonstrate both an increase of overall survival and a decrease of postoperative morbidity. A single randomized controlled trial has been conducted to investigate the effectiveness of neuronavigation in resecting intracerebral tumors: no rationale for the routine use of neuronavigation to improve the extent of resection and prognosis has been demonstrated. ${ }^{2}$ In the same way, the sole prospective randomized study using high-field intraoperative MRI in gliomas showed a significant increase of the extent of resection, but with nonetheless a high rate of new neurological deficits about $13 \%$.

The reason which could explain these disappointing results of image-guided glioma surgery is related to the fact 
that the brain itself has received less attention. Indeed, cerebral processing is traditionally conceived in a localizationist and rigid philosophy. By this principle, each region of the brain corresponds to a given function, and a lesion in a so-called "eloquent" area is therefore presumed to result in a massive and permanent neurological deficit. According to this view, a patient with a glioma in critical structure, e.g. Broca's area e for a long time wrongly considered as the area of speech ${ }^{4}$ e is usually not selected for surgical resection, in particular if the neurological examination is normal (for example after the diagnosis of a low-grade glioma following inaugural seizures). Recently, the concept of the brain connectome has emerged. Its goal is to capture the characteristics of spatially distributed dynamical neural processes at multiple spatial and temporal scales. Based upon this new science of "connectomics", which aims to map the neural connections, the central nervous system is an ensemble of complex distributed and parallel networks that form, reshape, and flush information dynamically, opening the door to a huge potential of post-lesional neuroplasticity.

Interestingly, awake mapping using direct electrical stimulation (DES) of the brain can be achieved in patients undergoing surgery for cerebral gliomas. In practice, patients perform several sensori-motor, visuospatial, language, cognitive or behavioral tasks while the surgeon temporarily disrupts discrete regions around or within the tumor, using DES. This electrical current is mimicking a genuine "virtual transient lesion", both at the level of cortex, and at the axonal level when the electrode is applied directly in contact with the white matter fascicles. Therefore, if the patient stops moving, speaking or produces wrong response, the surgeon avoid removing the stimulated site. In other words, DES is currently the sole method that permits a direct and real-time investigation of the individual functional anatomy of the brain. ${ }^{4}$ This information is very helpful, because cerebral tumors (especially slowgrowing disease as low-grade glioma) may generate mechanisms of functional reorganization, explaining why many patients experienced no or only slight neurological deficits at time of diagnosis. This means that there is a major interindividual anatomo-functional variability of the brain connectome, and that anatomic landmarks are definitely not reliable enough to operate a cerebral tumor. According to this new concept, the goal of glioma surgery is now to achieve a resection according to functional and not to oncological boundaries. Thus, the principle is to first understand the cerebral anatomo-functionalorganization for each patient, with the aim of removing a part of the brain invaded by a diffuse tumoral disease, on the condition nonetheless that this part of the brain can be functionally compensated e i.e. with no consequences on the quality of life. ${ }^{4}$

Interestingly, it has been shown in a recent metaanalysis of 8091 glioma patients, that functional mapping-guided resection using DES (a) identifies cortical and subcortical structures which are crucial for brain functions, allowing to perform surgeries in "eloquent" areas previously thought as "unresectable" thanks to potential of neuroplasticity (b) significantly reduces the risk of permanent postoperative neurological deficits about $3.4 \%$, including when operating in those "critical" structures (c) enables resections to be made according to functional limits, without a security margin around the eloquent structures, optimizing extent of resection and (d) therefore increasing overall survival. ${ }^{5}$ As a consequence, intraoperative DES is currently the standard of care for glioma surgery. It is also worth noting that DES mapping does not require additional costs to classical procedure, conversely to intraoperative imaging methods.

In summary, rather than expensive technological developments, such a conceptual shift with regard to cerebral connectomics, by switching from a modular to a delocalized view (in which brain functioning is underlain by large-scale and dynamic sub-networks processing together), has allowed an improvement of both extent of resection and quality of life in glioma patients who underwent intraoperative functional mapping using DES. Such a better knowledge of interactions between the natural history of gliomas (proliferation and migration) and brain adaptation (until the limits of neuroplasticity have been reached, then leading to the occurrence of neurological deficits) is a new way to solve the classical dilemma in glioma surgery e i.e. survival versus quality of life. It also opens the door to the concepts of "functional neurooncology" and of "preventive glioma surgery" based on the proposal of a screening policy for glial tumors, in order to achieve earlier and more complete resections while giving the opportunity to the patients to enjoy a normal life. Indeed, as already proposed in other organs, prophylactic surgical removal of brain glioma may now been considered in asymptomatic patients, due to the increased level of accuracy and reliability of the cognitive tasks performed in awake patients into the operating theater. Beyond movement and language, executive functions (as judgment or working memory), emotional aspects (as theory of mind and mentalizing) and even consciousness can be mapped by DES. ${ }^{4}$ This enables to tailor the extent of resection according to the individual distribution of the neural networks, resulting in the preservation of the quality of life defined for each patient e e.g. avoiding visual field deficit for a taxi driver, or calculation deficits in a mathematician, or disorders of complex bimanual coordination in a pianist, and so on. This paradigmatic shift based on the personalized study of the cerebral connectome (rather than on the use of expensive structural imaging technology in a systematic manner as if all brains were identical) has resulted in important medical applications in neurosurgery, neurology, neuro-rehabilitation and even in psychiatry; from a fundamental point of view, it has led to the proposal of new models in the field of cognitive neurosciences. ${ }^{4}$ Finally, because awake surgery with brain mapping can be performed everywhere in the world, it may also have economical implications. 


\section{Conflict of interest}

There are no known conflicts of interest associated with this publication and there has been no significant financial support for this work that could have influenced its outcome.

\section{References}

1. Pallud J, Audureau E, Blonski M, et al. Epileptic seizures in diffuse low-grade gliomas in adults. Brain 2014;137:449-62.
2. Willems PW, Taphoorn MJ, Burger H, Berkelbach van der Sprenkel JW, Tulleken CA. Effectiveness of neuronavigation in resecting solitary intracerebral contrast-enhancing tumors: a randomized controlled trial. J Neurosurg 2006;104:360-8.

3. Senft C, Bink A, Franz K, Gasser T, Seifert V. Intraoperative MRI guidance and extent of resection in glioma surgery: a randomised, controlled trial. Lancet Oncol 2011;12:997-1003.

4. Duffau H. Stimulation mapping of white matter tracts to study brain functional connectivity. Nat Rev Neurol 2015;11:255-65.

5. De Witt Hamer PC, Robles SG, Zwinderman AH, Duffau H, Berger MS. Impact of intraoperative stimulation brain mapping on glioma surgery outcome: a meta-analysis. J Clin Oncol 2012;30:2559-65. 